\title{
Pipe cooling perspectives for superconducting accelerating cavities
}

\author{
R. Ballantini, A. Chincarini, ${ }^{*}$ G. Gemme, and R. Parodi \\ Istituto Nazionale di Fisica Nucleare, via Dodecaneso 33, 16146 Genova, Italy
}

(Received 12 June 2003; published 29 August 2003)

\begin{abstract}
We explore the rf characteristics of pipe cooled superconducting cavities versus bath cooled ones, using different pipe configurations and different liquid helium temperatures. Pipe cooled cavities can perform nearly as well as bath cooled ones, provided a suitable pipe configuration and cavity wall thickness is chosen. Pure thermal estimates and fits with experimental data show that pipe cooling is a viable solution for future cavities.
\end{abstract}

DOI: 10.1103/PhysRevSTAB.6.083201

PACS numbers: 07.20.Mc, 41.75.Lx

\section{INTRODUCTION}

Superconducting rf cavities have been used in particle accelerators for several decades, these cavities being traditionally operated immersed in a liquid helium $(l \mathrm{He})$ bath. Nevertheless, several attempts have been made in the past to make use of the numerous operational and cost advantages of pipe cooling configuration: reduction in liquid helium inventory, minimized cooldown/warm-up times, and elimination of the $l \mathrm{He}$ vessel, which reduces the sensitivity to microphonics and provides easier access to all cavity components [1]. Furthermore, the fact that cavities are operated in pulse mode makes the pipe cooling scheme more attractive since it provides extra stiffening to the cell.

In this paper we compare the expected performances of bath cooled and pipe cooled single cells by means of a $2 \mathrm{D}$ computer code and we address the following questions:

(1) What kind of reduction in $Q_{0}$ values can we expect to have in a pipe cooled cavity? (2) What is the peak $B_{\text {surf }}$ field attainable if the losses were predominantly due to thermal effects? (3) If the losses in a bath cooled cavity were due to effects other than thermal (e.g., electron emission, multipacting, etc.), how would that cavity's performance be influenced by pipe cooling?

\section{MODEL}

Thermal behavior of cavities has been computed in various ways [2-4], mainly using one dimensional models. Despite several attempts, a coherent formulation that fits the measured data is still lacking, unless one uses $a d$ hoc parameters and functions added to the theoretical surface resistance.

This problem is mainly due to the fact that most of the $Q_{0}$ versus $E_{\text {acc }}$ measurements are affected by dissipation mechanisms that are not related to Ohmic losses alone, not to mention the field dependence effect of sputtered niobium films on copper [5].

Our approach is then twofold: first we consider a purely thermal problem, with no other dissipation mechanisms, and compare the efficiency of different pipe configurations and $l \mathrm{He}$ temperatures; then we take real measurements of the averaged surface resistance $\left\langle R_{\text {surf }}\right\rangle$ versus $E_{\text {acc }}$ in bath cooled cavities and use this curve to extrapolate the $\left\langle R_{\text {surf }}\right\rangle$ in the case of pipe cooling.

We carry out the calculation with the help of a $2 \mathrm{D}$ computer code that simulates the thermal behavior of axially symmetric structures, whose position dependent surface fields are given. The fields inside the structure come from OSCAR2D [6], a fast finite element code that computes all relevant rf quantities and field resonance distributions for axisymmetric cavities.

\section{A. Surface resistance}

When considering purely Ohmic dissipations, our calculations are based on an analytical model for the BCS resistance (Wilson formula), which has been proven to adequately match both the experimental data and the theoretical calculations [7]. The use of an analytical function has the advantage to considerably speed up the computation while preserving the correct parameters dependence. The function is

$$
R_{\mathrm{BCS}}=\left(\frac{f}{f_{0}}\right)^{2} \frac{\alpha}{T_{c r}-T_{\mathrm{int}}} \ln \left(\frac{\beta T_{\mathrm{int}} f_{0}}{f}\right) e^{-\Delta\left(T_{c r} / T_{\mathrm{int}}\right)} \sqrt{\cos \left[(\pi / 2)\left(T_{\mathrm{int}} / T_{c r}\right)^{2}\right]},
$$

where $\alpha, \beta$, and $\Delta$ are calculated to fit experimental data.

$T_{\text {int }}$ is the cavity's inner surface temperature in $K, f$ is the frequency in $\mathrm{Hz}$, and $f_{0}$ is the reference frequency at which experimental data are available. $T_{c r}$ is a function of

*Electronic address: andrea.chincarini@ge.infn.it the surface magnetic field $B_{\text {surf }}$ and, together with $T_{\text {int }}$, it is also a function of the position.

A small phenomenological residual resistance $R_{\text {res }}$, typically of the order of $10-50 \mathrm{n} \Omega$, is added and the overall position dependent surface resistance reads as follows: 


$$
R_{s}\left(\vec{r}, T_{\text {int }}, f, B_{\text {surf }}\right)=R_{\text {res }}+R_{\mathrm{BCS}}(T),
$$

where $\vec{r}$ indicates the position vector on the cavity inner surface, and $B_{\text {surf }}$ is the surface magnetic field in tesla.

Equation (2) though, once used to calculate the averaged $\left\langle R_{s}\right\rangle$, does not always correctly fit the experimental curves $\left\langle R_{S}\right\rangle$ versus $E_{\text {acc }}$ (or equivalently, versus peak $B_{\text {surf }}$ ). We took expression (2) only to look at the cavity from a purely Ohmic point of view, that is to study the cavity behavior in the hypothesis where the only limiting factors are the BCS surface resistance, the thermal conductivity of the material, and the way the cavity is cooled on the external surface.

Considering experimental data though, one sees that the cavity performances are often limited by other processes (like field emission, multipacting, or surface defects) much earlier than the predicted limit given by Eq. (2) [8].

Since our aim is to look for applicability of pipe cooling and not to justify experimental $\left\langle R_{s}\right\rangle$ curves, we can take the measured $\left\langle R_{s}\right\rangle$ curves for a bath cooled cavity as given effective residual resistance, that is

$$
R_{s}\left(\vec{r}, T_{\text {int }}, f, B_{\text {surf }}\right)=\tilde{R}_{\text {res }}\left(E_{\text {acc }}\right)+R_{\mathrm{BCS}}(T),
$$

where $\tilde{R}_{\text {res }}\left(E_{\text {acc }}\right)$ is a suitable function that fits the experimental data.

We noted though that, for the cases we have considered, the main contribution to the $\left\langle R_{s}\right\rangle$ came from field emission phenomena. It was therefore possible to use reasonable analytical functions to fit the measurements:

$$
\tilde{R}_{\mathrm{res}}=R_{\mathrm{res}}+\lambda_{1} E_{\mathrm{acc}}+\lambda_{2} E_{\mathrm{acc}}\left(\lambda_{3} E_{\mathrm{acc}}\right)^{5 / 2} e^{\left(-\lambda_{4} / \lambda_{3} E_{\mathrm{acc}}\right)},
$$

where $R_{\text {res }}=\lim _{E_{\text {acc }} \rightarrow 0}\left\langle R_{s}\right\rangle$ and $\lambda_{i}$ are free dimensional parameters.

The term multiplying $\lambda_{1}$ takes into account the linear trend observed in sputtered $\mathrm{Nb}$ on $\mathrm{Cu}$ cavities at low fields [5] while the term multiplying $\lambda_{2}$ is the Fowler-Nordheim term [8].

Once the $\lambda_{i}$ are found through a fit, Eqs. (3) and (4) are used in the thermal calculation for a bath cooled cavity and the results are checked to verify that they match the experimental data again. It is then easy to modify the external condition to account for pipe cooling.

$Q_{0}$ values are then calculated by

$$
Q_{0}=\frac{G_{k}}{\left\langle R_{s}\right\rangle},
$$

where $G$, in Ohm, is the geometry factor for the electromagnetic (e.m.) mode $k$, defined as

$$
G_{k}=\frac{\omega_{k} \mu_{0} \int_{V}|H|^{2} d v}{\int_{S}|H|^{2} d s} .
$$

\section{B. Thermal conductivity}

The thermal conductivities used in calculation come from experimental data [9], linearly interpolated and properly scaled for the residual resistivity ratio (RRR) values when needed. The different values of the thermal conductivity $\lambda$ used in the simulations have been computed scaling the measured curve $(R R R=60)$ according to the Wiedmann-Franz [10] law:

$$
\lambda(\text { new RRR })=\lambda(\operatorname{RRR}=60) \frac{\text { new RRR }}{60} .
$$

For $\mathrm{Nb}$ on $\mathrm{Cu}$ sputtered cavities, only $\mathrm{Cu}$ has been considered to account for heat transport, thus neglecting the sputtered $\mathrm{Nb}$ layer. Figure 1 shows both the $\mathrm{Nb}$ and $\mathrm{Cu}$ curves used in the model.

\section{Metal- $l$ He interface}

The power dissipated on the inner superconductor surface is transferred to the outer surface and then to the $l \mathrm{He}$ medium. Across the interface between a solid and $l \mathrm{He}$ there exists a temperature jump $\Delta T=T_{s}-T_{B}$, where $T_{s}$ is the solid outer surface temperature and $T_{B}$ is the bath temperature (both in K). $\Delta T$ is related to the heat flux $q_{e}$ in $\mathrm{Wm}^{-2}$ across the interface so that $T_{s}$ can be described by the following relation [3]:

$$
T_{s}= \begin{cases}\left(T_{B}^{4}+4 q_{e} R_{k} T_{B}^{3}\right)^{1 / 4} & T_{B} \leq 2.18 \mathrm{~K}, \\ T_{B}+\left(\frac{q_{e}}{h}\right)^{1 / a} & 20.18<T_{B} \leq 4.2 \mathrm{~K} .\end{cases}
$$

This expression shows that $\Delta T$ is also dependent on the $l$ He regime: for superfluid $l \mathrm{He}\left(T_{B} \leq 2.18 \mathrm{~K}\right), T_{s}$ is driven by the Kapitza resistance $R_{k}$ that, for chemically polished and annealed $\mathrm{Nb}$, has been measured to be [11]:

$$
R_{k}^{-1}=(0.020 \pm 0.003) 10^{4} T^{(4.65 \pm 0.28)}
$$

in $\mathrm{W} \mathrm{m}^{-2} \mathrm{~K}^{-1}$. Recent measures [12] report generally higher values for the Kapitza conductance, especially

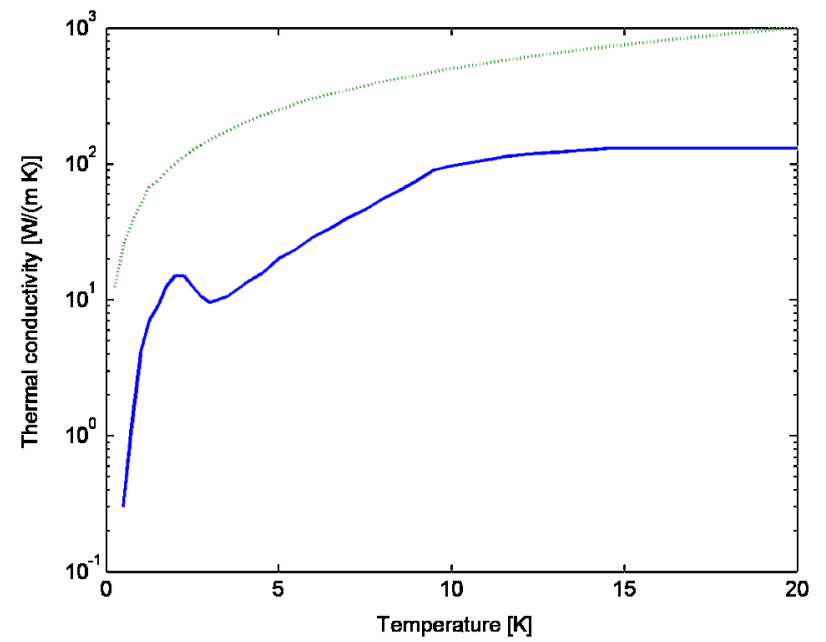

FIG. 1. (Color) Experimental thermal conductivity for $\mathrm{Nb}$ (solid line) and $\mathrm{Cu}$ (dashed line). $\mathrm{Nb}$ data refer to a $\mathrm{RRR}=$ 60 sample. 
for chemically treated surfaces. The values in Eq. (8) are therefore quite conservative.

For the $l \mathrm{He}-\mathrm{I}$ regime, $T_{s}$ is proportional to a power of $q_{e}$, whose dimensional parameters have been experimentally determined to be $h \approx 1.23 \times 10^{4}$ and $a \approx 1.45$ [13].

Equation (7) holds below a critical heat flux $(\approx$ $10^{4} \mathrm{~W} \mathrm{~m}^{-2}$ ) at which film boiling sets on and the heat transfer rate sharply decreases by approximately 1 order of magnitude.

Experimental data for the critical heat flux show a rather large scattering and are influenced by several factors such as the specimen geometry, submersion depth, and $l \mathrm{He}$ bath temperature. The value of $10^{4} \mathrm{~W} \mathrm{~m}^{-2}$ is often assumed for He-II constrained in long tubes and we have taken this conservative approach in spite of the fact that critical fluxes up to $6 \times 10^{4} \mathrm{~W} / \mathrm{m}^{2}$ have been observed [14].

\section{Thermal modeling}

Our simulator computes the temperature profile as well as other relevant quantities of an axially symmetric structure whose e.m. fields distribution and properties are given.

The structure symmetry allows a $2 \mathrm{D}$ simulation and integrates seamlessly with the e.m. computation from OSCAR2D (see Fig. 2).

Figure 3 sketches the elementary surface elements on which the calculation is carried. $s_{i}, s_{o}, s_{r}$, and $s_{t}$ are surface elements at the curvilinear coordinate $l$, referring, respectively, to the inner surface (source of the rf heating), the outer surface (in contact with the bath), and the right and left surfaces, where lateral heat conduction takes place.

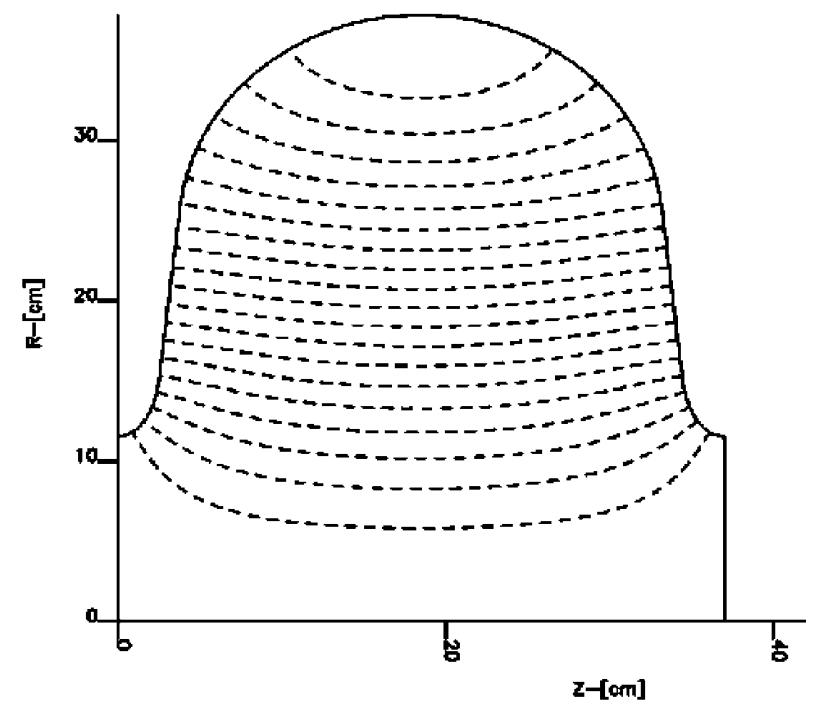

FIG. 2. Electric field lines for the $\mathrm{TE}_{011}$ mode in a single cell. Graphical output by OSCAR2D.

\section{LHe}

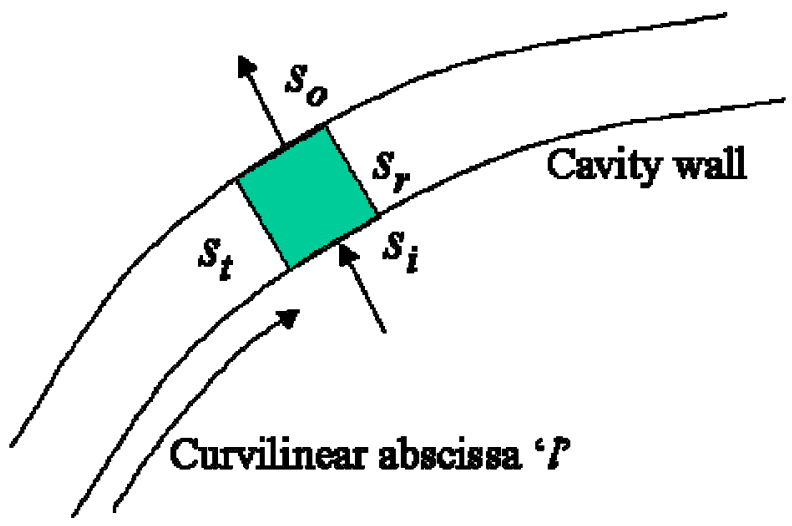

FIG. 3. (Color) Sketch of the volume element used for the temperature modeling.

The simulation starts with both the surfaces $\left\{s_{i}\right\}$ and $\left\{s_{o}\right\}$ at the bath temperature and then, for each field value, calculates iteratively until convergence is reached.

In the case of pipe cooling, the outer surface element $s_{o}(l)$ which is not in contact with a pipe, is bound to reach the same temperature of the corresponding $s_{i}(l)$ and the heat is therefore dissipated only through $s_{r}(l)$ and $s_{t}(l)$.

Cavity thickness, material properties, and e.m. configuration as well as all relevant parameters can be varied at will.

\section{RESULTS}

\section{A. Thermal effects}

This simulation set compares the expected results for a bath cooled versus a pipe cooled cavity when only BCS surface resistance and the material thermal conductivity are taken into account.

Figures 4 and 5 show, respectively, the results for a TRASCO $\beta=0.85 \mathrm{Nb}-\mathrm{Cu}$ cavity and for a TRASCO $700 \mathrm{MHz} \mathrm{Nb}$ bulk cavity, both operated on the $\mathrm{TM}_{010}$ [15-17].

As expected, the maximum $B_{\text {surf }}$ predicted for bath cooling is much higher than the experimental values, thanks to the lack of other dissipation mechanisms. Pipe cooling performances are significantly different only in the high field region and exhibit almost no $Q_{0}$ degradation in the range $0-80 \mathrm{mT}\left(T_{l \mathrm{He}}=1.8 \mathrm{~K}\right)$ and in the range $0-25 \mathrm{mT}\left(T_{l \mathrm{He}}=4.2 \mathrm{~K}\right)$.

The pipe scheme used for one of these simulations is sketched in Fig. 6, where the pipes position is plotted versus the curvilinear abscissa running on the cavity's profile.

The normalized $B$ profile pictured in Fig. 6 refers to the TRASCO $\beta=0.85$ cavity. For this test, only three pipes of approximately $6 \mathrm{~cm}$ in diameter are used to cool the cavity, and they have been positioned in the highest $B$ 


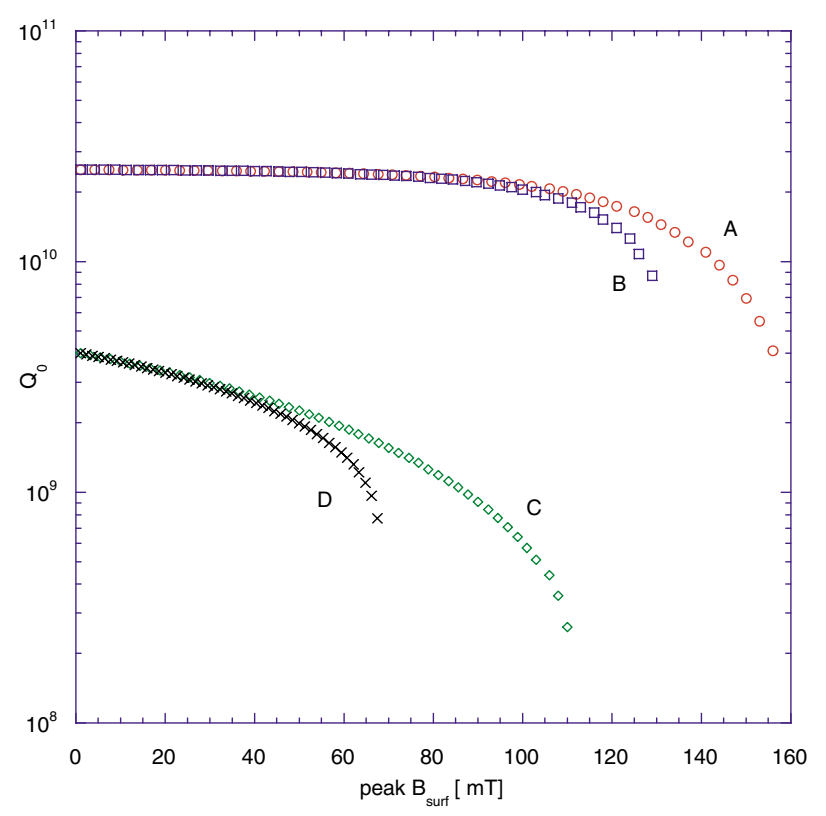

FIG. 4. (Color) Performance comparison computed for a TRASCO $\beta=0.85$ cavity. Simulation parameters are $f=$ $343 \mathrm{MHz}, G=250 \Omega, R_{\text {res }}=10 \mathrm{n} \Omega, C u$ thickness $=6 \mathrm{~mm}$, and $\mathrm{RRR}=275$. $(A)$ and $(B)$ are, respectively, bath and pipe cooled at $T_{l \mathrm{He}}=1.8 \mathrm{~K},(C)$ and $(D)$ are bath and pipe cooled at $T_{l \mathrm{He}}=4.2 \mathrm{~K}$.

field region yielding a cavity surface coverage of approximately $17 \%$.

We can also study the effect of different pipe cooling schemes, so that the best configuration can be chosen. The

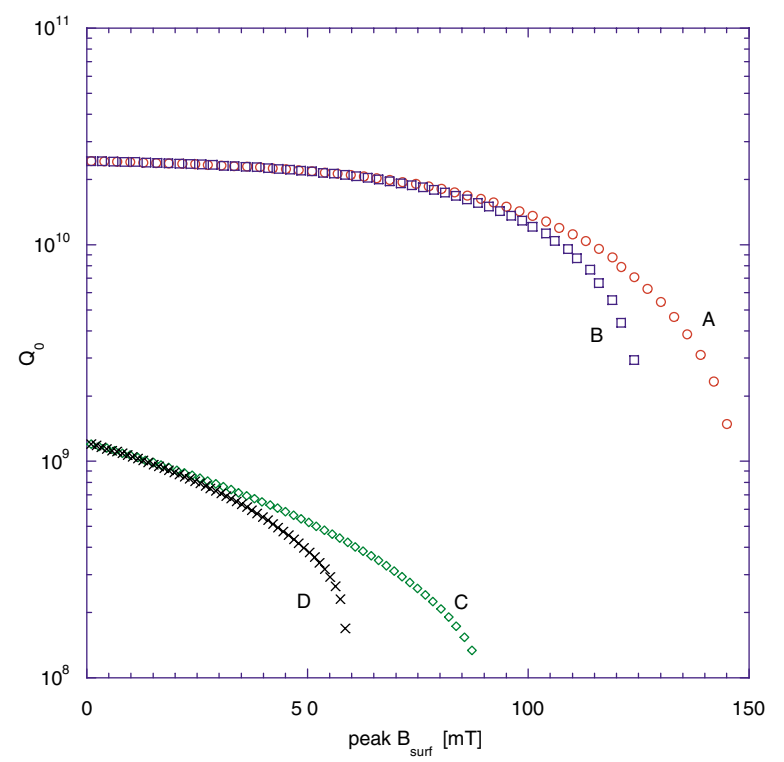

FIG. 5. (Color) Performance comparison computed for a TRASCO $700 \mathrm{MHz}$ cavity. Simulation parameters are $f=$ $707 \mathrm{MHz}, G=250 \Omega, R_{\text {res }}=10 \mathrm{n} \Omega, C u$ thickness $=4 \mathrm{~mm}$, and $\mathrm{RRR}=275$. $(A)$ and $(B)$ are, respectively, bath and pipe cooled at $T_{l \mathrm{He}}=1.8 \mathrm{~K},(C)$ and $(D)$ are bath and pipe cooled at $T_{l \mathrm{He}}=4.2 \mathrm{~K}$.

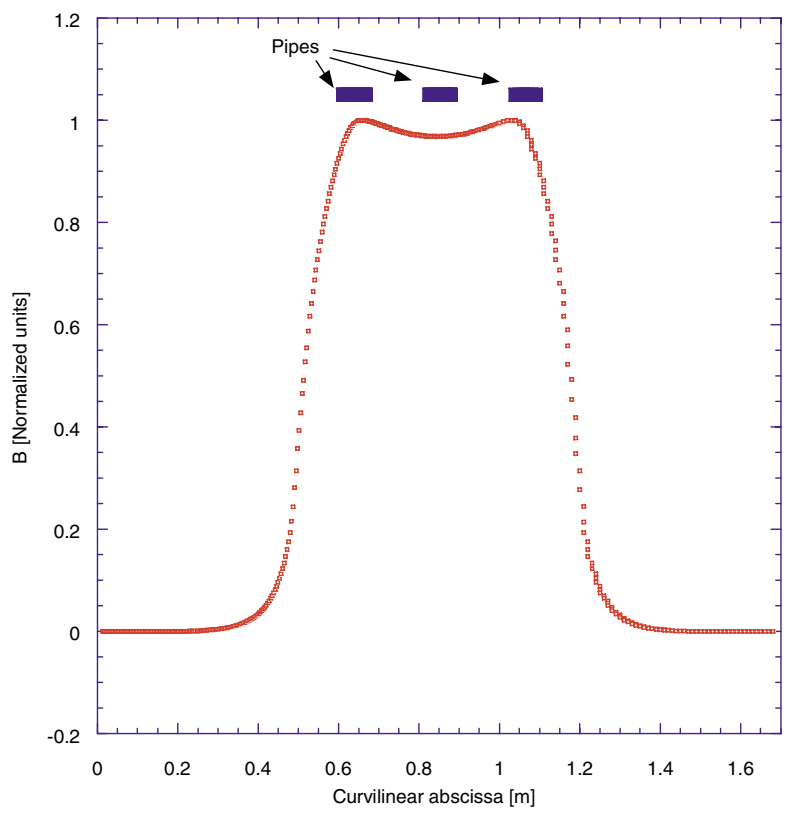

FIG. 6. (Color) Pipes position relative to the magnitude of the magnetic field on the surface. Each pipe has a diameter of approximately $6 \mathrm{~cm}$.

configurations share approximately the same coverage on the cavity surface $(\approx 20 \%$ ), their difference consisting only in the number of pipes and their distribution.

Figure 7 shows the results of this test for a $707 \mathrm{MHz}$ cavity at $4.2 \mathrm{~K}$. The choice in the cooling schemes does not yield an appreciable difference in the $Q_{0}$ curves unless one wants to run the cavity near the (theoretical) transition point.

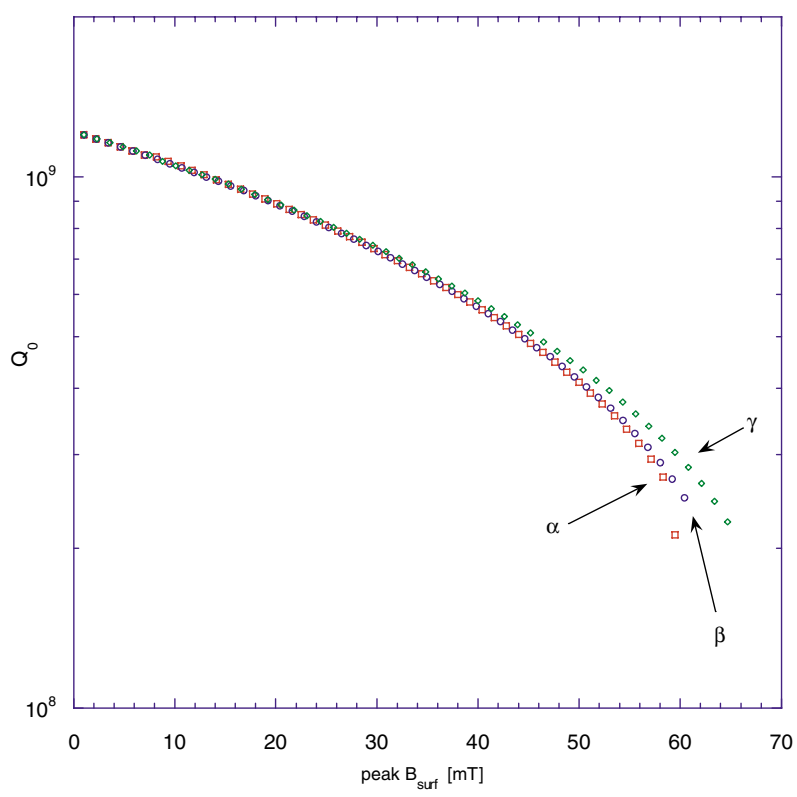

FIG. 7. (Color) Simulation of a TRASCO $700 \mathrm{MHz}$ cavity at $4.2 \mathrm{~K}$ with pipe cooling. The cavity surface coverage is $20 \%$ for all curves. Curve $(\alpha): 3$ pipes $\varnothing=1.8 \mathrm{~cm}$; curve $(\beta)$ : 5 pipes $\varnothing=1.0 \mathrm{~cm}$; curve $(\gamma)$ : 9 pipes $\varnothing=0.7 \mathrm{~cm}$. 


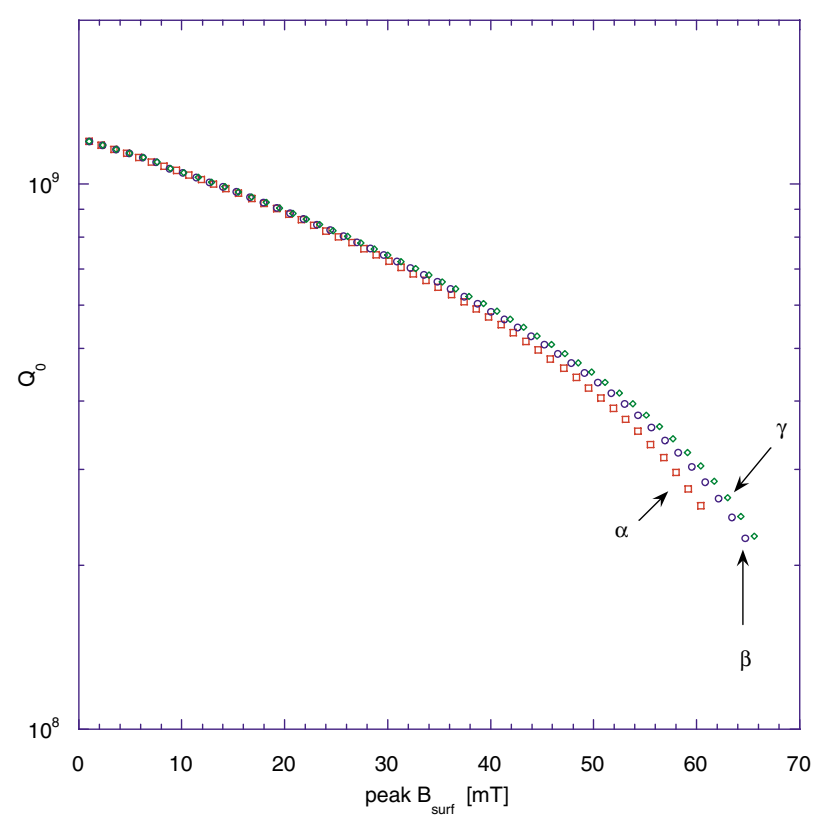

FIG. 8. (Color) Simulation of a TRASCO $700 \mathrm{MHz}$ cavity at $4.2 \mathrm{~K}$ with pipe cooling. The cavity is covered with nine pipes for a total of $20 \%$ of its surface. The curves relate to different $\mathrm{Cu}$ thickness. Curve $(\alpha)$ : thickness $=2 \mathrm{~mm} ;$ curve $(\beta)$ : thickness $=6 \mathrm{~mm}$; curve $(\gamma)$ : thickness $=10 \mathrm{~mm}$.

A slight improvement, which favors a greater number of pipes, is nevertheless observable. Similarly, a thicker cavity wall improves the stability as shown in Fig. 8.

These results support the idea that pipe cooling is a viable solution at least in the case where no dissipation mechanisms other than thermal breakdown are allowed. They also show that there is a non-negligible freedom in choosing the pipe distribution, provided enough surface coverage exists in the high field region.

The simulations discussed above however, do not include pointlike surface defects.

\section{B. Frequency and RRR dependence}

Pipe cooling efficiency can also be tested as a function of several cavity parameters. We have run some simulations to study the thermal behavior dependence on the cavity's frequency and the Nb RRR.

Figure9 shows the $Q_{0}$ performances with several RRR values and refers to a TRASCO $700 \mathrm{MHz}$ cavity cooled at $T_{l \mathrm{He}}=1.8 \mathrm{~K}$ with the help of three pipes of diameter $\varnothing=$ $2.2 \mathrm{~cm}$, for a surface coverage of $\approx 23 \%$.

It may be of interest to look at the relative performance of a pipe cooled cavity versus a bath cooled one as function of the RRR. We have arbitrarily chosen to define this relative performance with the help of two parameters:

$$
\theta_{f}=\frac{\left.\max B\right|_{\text {pipes }}}{\left.\max B\right|_{\text {bath }}}, \quad \theta_{q}=\frac{\left.\Delta Q_{0}\right|_{\text {bath }}}{\left.\Delta Q_{0}\right|_{\text {pipes }}} .
$$

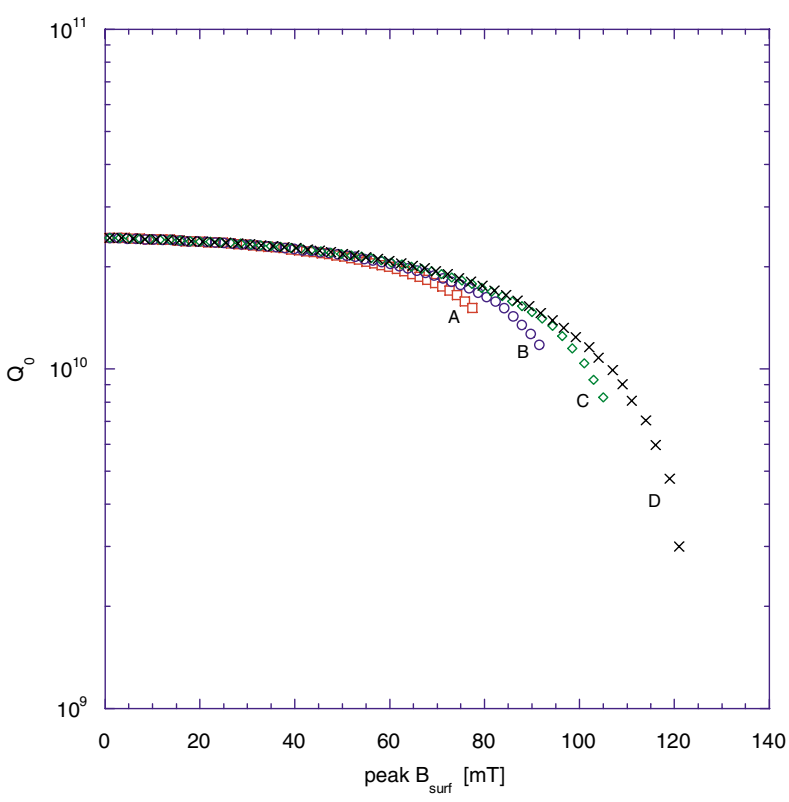

FIG. 9. (Color) Performances of a three-pipes cooled, $700 \mathrm{MHz}$ single cell, with different $\mathrm{Nb}$ purity. Curve $(A)$ is for a $\mathrm{RRR}=$ $50,(B)$ for a $\mathrm{RRR}=100,(C) \mathrm{RRR}=250$, and $(D) \mathrm{RRR}=500$.

$\theta_{f}$ is a measure of how well the pipe cooled cavity is able to sustain high intensity fields. $\theta_{q}$ is the relative $Q_{0}$ slope ratio, averaged over the lowest field range, that is over the range $\left[0,\left.\max B\right|_{\text {pipes }}\right]$. Ideally, $\theta_{q}$ should approximate 1 , meaning that the $Q_{0}$ slope (versus $B$ ) for the pipe cooled cavity is comparable to the bath cooled one. A low value for both parameters indicates a low relative performance.

Since the pipe cooled cavity performances are always lower than the bath cooled counterparts, we have $0<$ $\theta_{f}, \theta_{q}<1$.

Figure 10 shows these parameters as a function of the RRR, whose values (from 10 to 1000), although somewhat extreme, have been used to show the extent of the differences. Figure 10 confirms the need for a high thermal conductivity (RRR $>200)$ and shows that extreme values do not appreciably increase the stability.

Frequency dependence has been tested by scaling the linear dimensions of a $700 \mathrm{MHz}$ cavity in order to have its resonant frequency going from $500 \mathrm{MHz}$ up to $3 \mathrm{GHz}$, all cavities cooled at $T_{l \mathrm{He}}=1.8 \mathrm{~K}$.

It is important to note that we have scaled the cavity linear dimensions and tube diameters (in order to keep the surface coverage a constant), whereas the wall thickness has been kept unchanged $(2 \mathrm{~mm})$.

All other parameters such as residual resistance, geometry factor, and $l \mathrm{He}$ temperature have been kept constant throughout all computations.

We have simulated the cooling using three pipes, whose diameters ranged from $3.1 \mathrm{~cm}$ (for the $500 \mathrm{MHz}$ cavity) to $5 \mathrm{~mm}$ ( $3 \mathrm{GHz}$ cavity), keeping the surface coverage at $24 \%$. Figure 11 shows the $Q_{0}$ performances for the cavity scaled to operate at $500 \mathrm{MHz}$ and $3 \mathrm{GHz}$. 


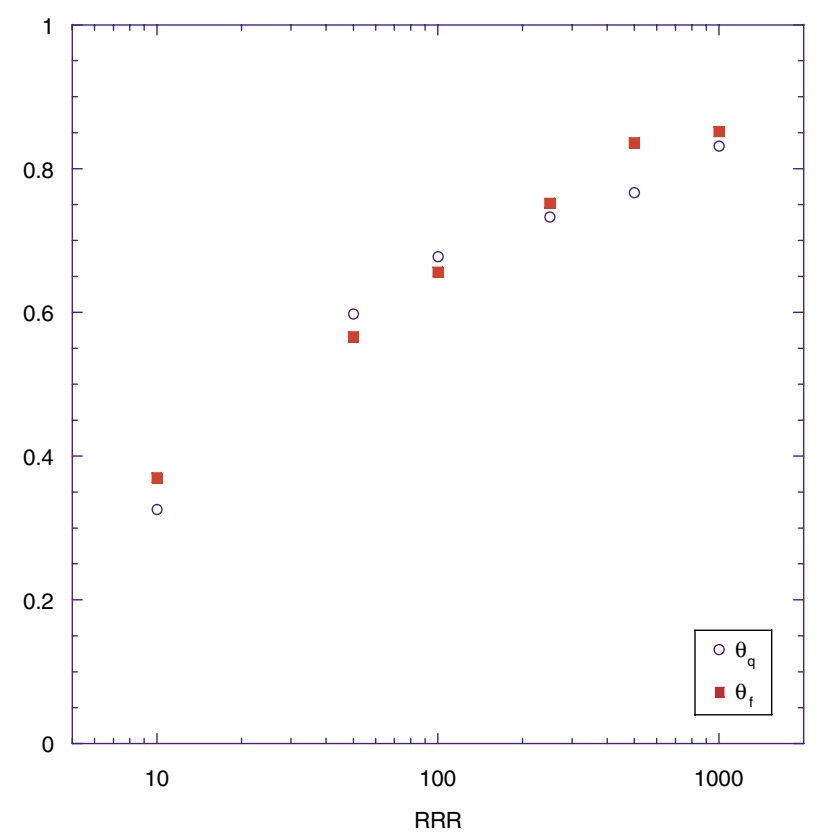

FIG. 10. (Color) Relative performance of a $700 \mathrm{MHz}$ cavity as a function of the $\mathrm{Nb}$ RRR.

Figure 12 shows the relative performance parameters versus the cavity frequency. Interestingly, the relative performances do not change appreciably in a quite wide frequency range. This means that, when pipe cooled, one could expect to achieve a maximum field of about $80 \%$ of the field attainable in a bath cooled cavity, almost regardless of the operating frequency.

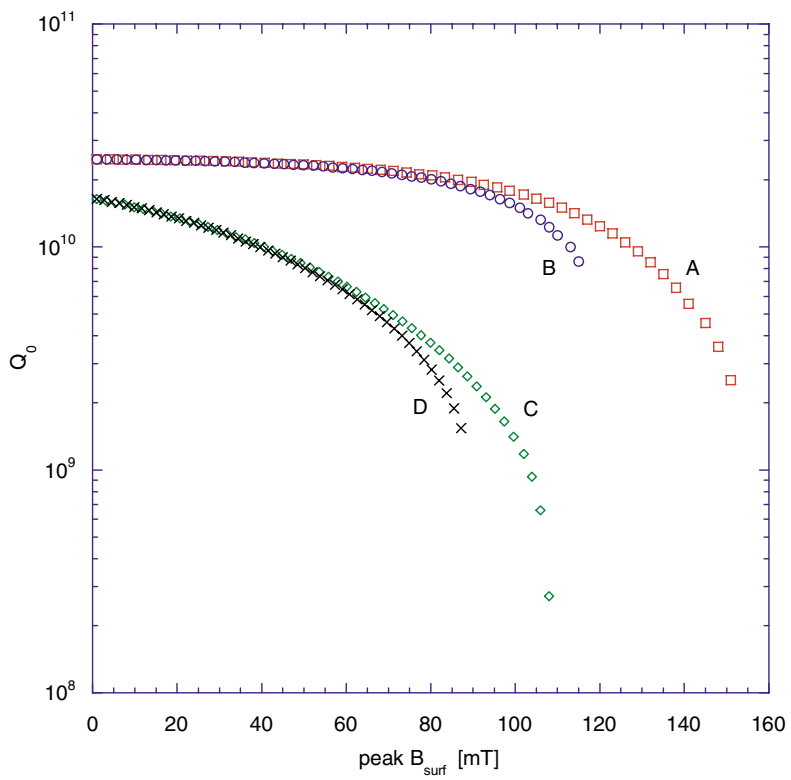

FIG. 11. (Color) Performance comparison for a $2 \mathrm{~mm}$ thick, bulk $\mathrm{Nb}$ cavity whose dimensions were scaled to operate at $500 \mathrm{MHz}$ and $3 \mathrm{GHz}$. RRR $=300$ for all curves. Curve $(A)$ : bath cooled, $500 \mathrm{MHz}$; $(B)$ : pipe cooled, $500 \mathrm{MHz} ;(C)$ : bath cooled, $3 \mathrm{GHz}$; $(D)$ : pipe cooled, $3 \mathrm{GHz}$.

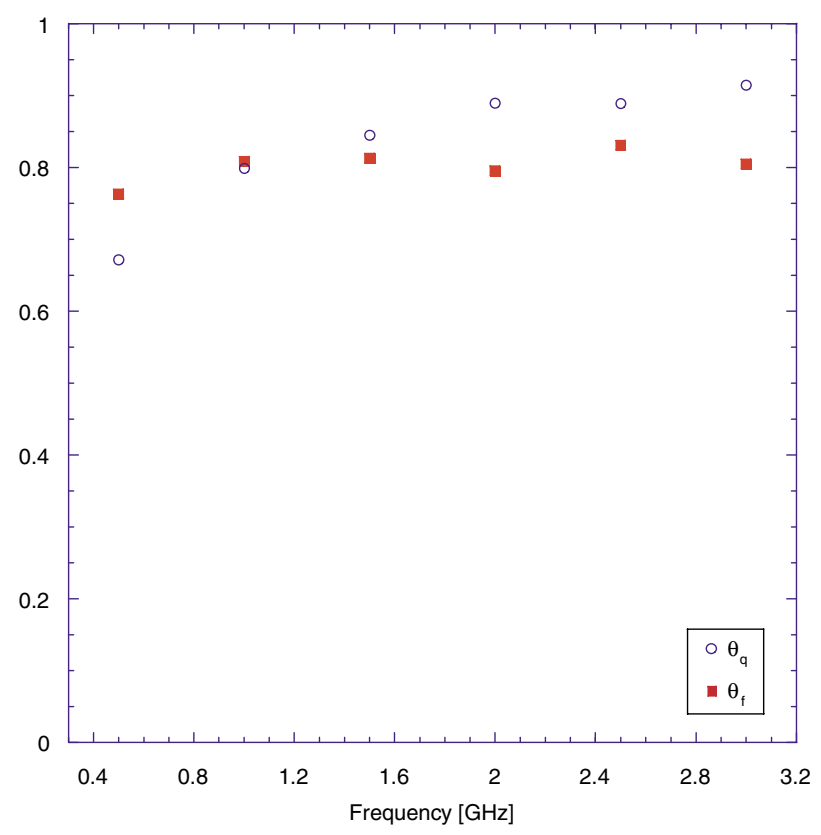

FIG. 12. (Color) Relative performance parameters as a function of the cavity frequency. Bulk $\mathrm{Nb}$ cavity, $2 \mathrm{~mm}$ thick, $\mathrm{RRR}=300$, three pipes cooling.

These results obviously are valid for a very ideal situation, especially regarding the cavity surface loss mechanism. Furthermore, keeping the thickness of the cavity as a constant over the whole frequency range, for example, would pose serious mechanical problems at low frequency. Similarly, scaling the pipe diameters to $5 \mathrm{~mm}$ may be unpractical.

\section{Surface defects}

One of the few published results of pipe cooled cavities versus bath cooled ones was given by Susta et al. in 1993 [1]. In their paper, they showed a very nice curve of $Q_{0}$ versus peak $E_{\text {surf }}$ in a bath cooled, bulk Nb cavity, accompanied by a pipe cooled measure, which suffered a reduction for peak $E_{\text {surf }}$ of approximately $47 \%$ (peak $E_{\text {surf }} \approx 57 \mathrm{MV} / \mathrm{m}$ for bath cooling, peak $E_{\text {surf }} \approx$ $30 \mathrm{MV} / \mathrm{m}$ for pipe cooling, $\left.T_{l \mathrm{He}}=1.8 \mathrm{~K}\right)$. In Kneisel's paper, the cavity was cooled through a single, large pipe (71 mm wide) welded on the cell together with two other pipes welded on either side of the drift tubes.

To check for a qualitative agreement with their experimental results, we have performed a simulation using their same pipe scheme and we have also added the simulation equivalent of a surface defect.

In our code the surface defect can only be simulated by changing the properties of an annular surface, whose typical dimensions are $40 \mathrm{~mm}$ in radius and $1 \mathrm{~mm}$ in height.

The results are shown in Fig. 13. The qualitative agreement with the measure (not reported here) reveals that the presence of a surface defect can be a valid explanation for 


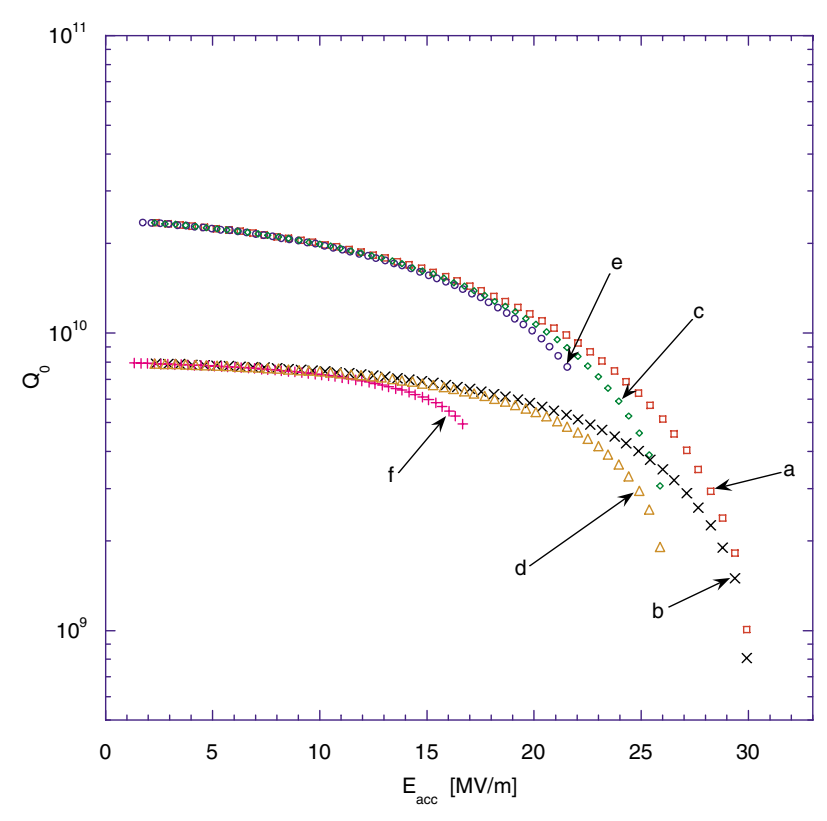

FIG. 13. (Color) Surface defect simulation for a $1500 \mathrm{MHz}$ cavity. Curves are labeled as follows: (a) bath cooled, no surface defect; $(b)$ bath cooled with surface defect; $(c)$ five pipes $\varnothing \approx 2 \mathrm{~cm}$, surface coverage $38 \%$, no defect; (d) same as (c) but with surface defect; $(e)$ single pipe $\varnothing \approx 7.1 \mathrm{~cm}$, surface coverage $38 \%$, no defect; $(f)$ single pipe with surface defect.

the severe loss in performance $(\approx 44 \%$ reduction on the maximum attainable $E_{\text {acc }}$ field).

The simulation also shows that in the bath cooled case the presence of the defect does not deteriorate the achievable peak surface fields [curves $(a)$ and $(b)$ in Fig. 13]. This means that the cavity may perform very well in the bath and yet may turn out to be strongly affected by the defect when pipe cooled [curves $(b)$ and $(f)$.

The surface defect though, whose presence was taken into account in the original paper as well, is not the only cause responsible for the cavity behavior. A wiser choice for the pipes distribution could have helped; that is, a greater number of pipes suitably distributed perform better than a single big pipe, even if the single pipe surface coverage is a little greater than the multipipe configuration.

A configuration involving several pipes is therefore more forgiving in case of a surface bad spot [compare curves $(c),(d)$, and $(f)$ in Fig. 13].

\section{Measurement fits}

Real cavities performance curves often exhibit signatures of effects like electron field emission and multipacting. Because of their variability in strength and occurrence, it is difficult to account for them in a model.

In this paper we have considered a set of measurements on a TRASCO $700 \mathrm{MHz}$ cavity performed at Saclay, France [18].

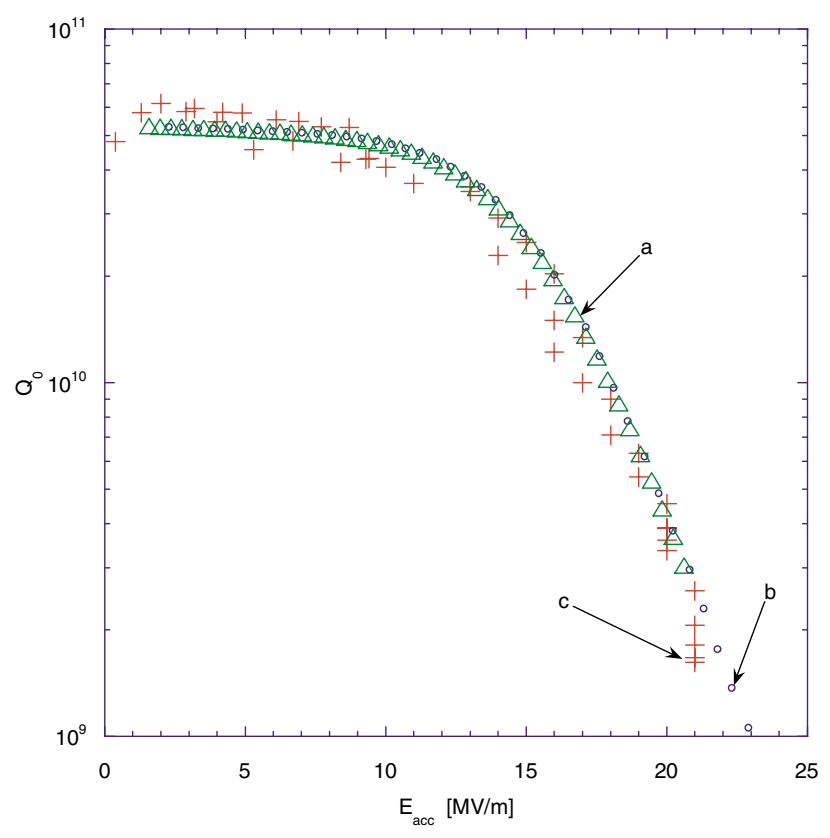

FIG. 14. (Color) Performance simulation and experimental data for a $700 \mathrm{MHz}$ cavity suffering from electron field emission. $(a)(\triangle)$ pipe cooled simulation; $(b)$ (o) bath cooled simulation; $(c)(+)$ measured data.

These measurements clearly show a $Q_{0}$ value degradation due to field emission, therefore we have used Eqs. (3) and (4) to fit the effective residual resistance, where the Fowler-Nordheim term accounts for the sharp increase of $\left\langle R_{s}\right\rangle$ at relatively low accelerating fields.

Figure 14 shows the measured data, the simulation for the bath cooled cavity, and the expected performance of the pipe cooling configuration on the same plot. In this test, we have simulated three pipes of approximately $1.5 \mathrm{~cm}$ in diameter equally spaced around the maximum value of the surface magnetic field.

If we restrict our analysis to relatively low $E_{\text {acc }}$ values $(<20 \mathrm{MV} / \mathrm{m})$, pipe cooling does not have any appreciable effect. The difference lies in the abrupt transition that takes place at a far lower field than the expected bath cooled counterpart $(\approx 20 \mathrm{MV} / \mathrm{m}$ for pipe cooling, $\approx$ $28 \mathrm{MV} / \mathrm{m}$ for bath cooling).

The simulations we performed, compared to the available real data on cavities limited by field emission, show that pipe cooling should not alter the overall cavity performance.

Electron emission normally occurs at field's values far lower than the one needed for thermal breakdown. It is therefore reasonable that, for those field's values, pipe cooling should perform nearly as well as bath cooling.

\section{APPLICABILITY}

Pipe cooling can also have interesting applications outside the world of particle accelerators. As an 


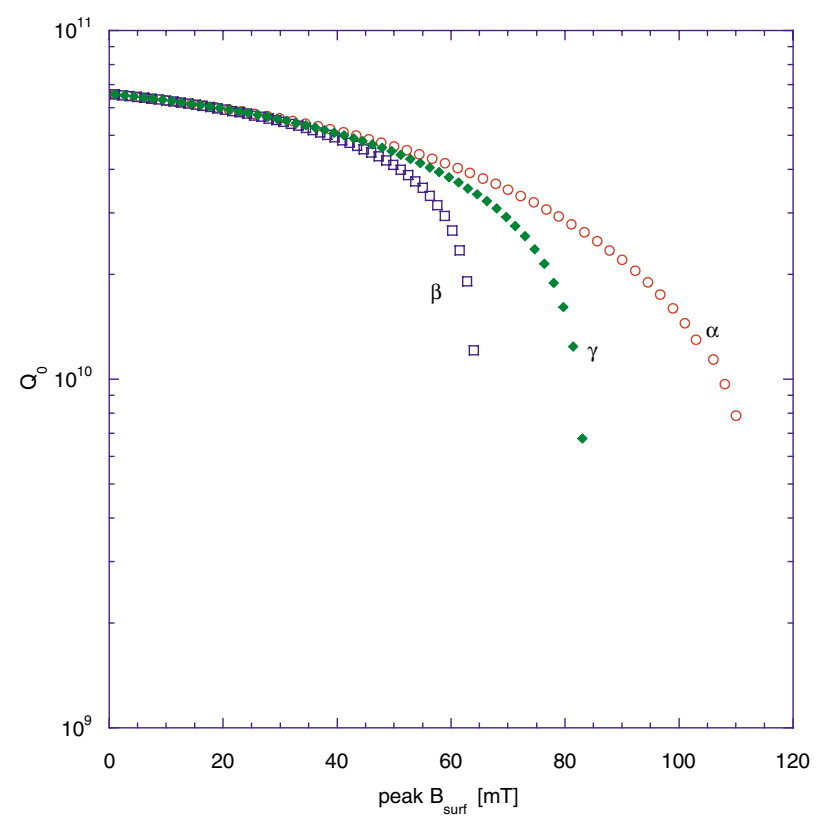

FIG. 15. (Color) $2 \mathrm{GHz}$ double spherical cell cavity. Curve $(\alpha)$ : $2 \mathrm{~mm}$ bulk Nb , bath cooled. Curve $(\beta): 2 \mathrm{~mm}$ bulk $\mathrm{Nb}$, pipe cooled with 2 pipes $\varnothing=3 \mathrm{~cm}$, cavity surface coverage $\approx 18 \%$. Curve $(\gamma)$ : $\mathrm{Nb}$ on $\mathrm{Cu}$ (5 mm thick), pipe cooled with the same configuration as in $(\beta) . T_{l \mathrm{He}}=1.8 \mathrm{~K}$ for all curves.

example, we report the use of superconducting cavities for gravitational waves detection [19]. This detector uses two nearly spherical cells coupled through a variable length tube and operated on the $\mathrm{TE}_{011}$ mode.

For this purpose, in contrast to accelerator applications, the spherical cells need to have a very high mechanical quality factor $Q_{\text {mech }}$, at least for one of the quadrupolar mechanical modes of resonance.

Since initial estimates suggest that bath cooling may impair the mode's $Q_{\text {mech }}$, pipes can be electron beam welded on the cell in such a way as to minimally perturb the quadrupolar mode oscillation. That is, pipes should run on the nodal lines of the mechanical resonant mode.

For this particular application, we have calculated the $Q_{0}$ estimates considering purely thermal dissipations, that is, with no surface defects and no electron emission-the latter assumption is justified by the $\mathrm{TE}_{011}$ mode of resonance.

The results are sketched in Fig. 15 and show a quite dramatic decrease in the theoretical performance (compared to the bath cooled case). This is due to the nonoptimal positioning of the pipes with respect to the e.m. field distribution and to the use of only two small pipes, both characteristics chosen to cope with the quadrupolar nodal lines.

As shown in the same figure, performances can be considerably improved using $\mathrm{Nb}$ on $\mathrm{Cu}$ cavities with a $\mathrm{Cu}$ thickness of approximately $5 \mathrm{~mm}$.

\section{CONCLUSION}

Bulk niobium and $\mathrm{Nb}-\mathrm{Cu}$ technologies are nowadays quite mature. We can therefore envisage an improvement in terms of design freedom and cost management for pipe cooled structures.

The remark that real pipe cooled cavities are much more sensitive to any nonideality, due to their lessened heat dissipation capabilities, is generally true. Nevertheless, the cavity behavior can be tamed by properly choosing the pipes size and distribution.

The performance estimations presented in this paper strongly support the call for a more experimental setup implementing pipe cooling.

[1] J. Susta, P. Kneisel, and M. Wiseman, in Proceedings of the International Conference on Particle Accelerator, Washington, DC, 1993 (IEEE, Piscataway, NJ, 1993), p. 1060.

[2] H. Vogel, DESY Laboratory Report No. DESY M-87-04, 1987.

[3] P. Fernandes and R. Parodi, Cryogenics 11, 433 (1984).

[4] R. L. Geng, in Proceedings of the International Conference on RF Superconductivity, 2001, Tsukuba (proc. ref. 010212-01).

[5] C. Benvenuti et al., Physica (Amsterdam) 316C, 153-188 (1999).

[6] P. Fernandes and R. Parodi, in Proceedings of the LINAC 86, Stanford, CA (SLAC Report No. 303, 1986), p. 330.

[7] P. B. Wilson, SLAC Laboratory Report No. SLAC-TN70-35, 1970.

[8] H. Padamsee, J. Knobloch, and T. Hays, $R f$ Superconductivity for Accelerators (Wiley, New York, 1998).

[9] NBS monograph.

[10] M.W. Zemansky, Heat and Thermodynamics (McGrawHill, New York, 1968), p. 94.

[11] K. Mittag, Cryogenics 13, 94 (1973).

[12] A. Boucheffa and M. X. Francois, in Proceedings of the Seventh Workshop on RF Superconductivity, Gif sur Yvette, France, 1995, pp. 659-669.

[13] C. Johannes, in Proceedings of the International Conference on Cryogenic Engineering, ICEC 3, Berlin, Germany, 1970, p. 97.

[14] D. N. Lyon, Int. Adv. Cryog. Eng. 14, 159 (1969).

[15] http://trasco.lnl.infn.it/

[16] D. Barni, in Proceedings of the International Conference on RF Superconductivity, 2001, Tsukuba (JP) (proc. ref. TA009).

[17] R. Parodi, in Proceedings of the International Conference on RF Superconductivity, 2001, Tsukuba (JP) (proc. ref. PR005).

[18] H. Safa, in Proceedings of the International Conference on RF Superconductivity, 2001, Tsukuba (JP) (proc. ref. MA008).

[19] A. Chincarini et al., Classical Quantum Gravity 20, 3505-3522 (2003). 